\title{
A UNIVERSAL MICROPHOTOMETER AND SOLAR CORONA POLARIMETRY
}

\author{
P. KOTRČ ${ }^{1}$, M. KNIZŽEK ${ }^{1}$ and J. SÝKORA ${ }^{2}$ \\ ${ }^{1}$ Astronomical Institute, 25165 Ondrejov, Czech Republic \\ ${ }^{2}$ Astronomical Institute, 05960 Tatranska Lomnica, Slovak Republic
}

Realization of solar corona polarimetry pertains to astrophysical centre-symmetrical problems. Usual ways of digitization are not optimum for this task. Coronal images obtained during solar eclipse at different angles of the polarizer should be digitized symmetrically in relation to the centre of the solar disk. Unfortunately, digitization of such centre-symmetrical images is usually performed by rectangular scanning with a firmly oriented digitization slit. Corresponding pixels in different images have to be found by methods of shifting the centres of the relevant images and a necessary rotation of the images. When the images are almost identical, or at least very similar each to other, the final fitting of the images can be tested by calculating the best correlation. As a rule, this is not the case for solar corona images obtained with different angle of the polarizer. In addition, after rotation of the digitized image, the digitisation rectangular networks become incompatible and thus some information is changed or even lost.

To avoid these complications in processing of the centre-symmetric corona images, we used the universal microphotometer (Zicha et al. 1992) in the mode of scanning in the polar coordinates system. We have processed the images from the total solar eclipse observed on July 11, 1991 (La Paz, Mexico). The observation and photographic records of the linearly polarized white-light corona and those in the green spectral line FeX $I V 530.3 \mathrm{~nm}$ are described by Sykora \& Badalyan (1992).

A special procedure for finding the centre of the solar disk on each image was used. A film strip with the image was put on the microphotometer table in a fixed orientation of the solar image to the coordinate axes of the table. The orientation has to be identical for all series of exposures. The centre of the Moon was then precisely found using about 8 points at its circular periphery by the least squares method. Evaluated differences of coordinates between the lunar and solar centres for each time of observation were inserted and a new beginning of the table coordinate system with its centre identical with the solar disk centre was set. Then the scanning of the solar corona image in polar coordinates was begun, while the position angle of the slit was permanently controlled and adjusted to be tangential to the solar radius at each position. As the relevant pictures were measured at the same orientation, the corresponding pixel values were located at the same positions $a_{i j}$ in the digitization matrices. The intensity values in corresponding pixels of relevant images can be easily processed to evaluate the real corona brightness as well as the degree and angle of its polarization.

Principal advantages of the digitisation measuring process performed with a rotating slit in polar coordinate system, when the beginning of coordinate system is identical with the solar disk 
centre, are as follows:

1) the rotating slit of the microphotometer moving along the radials in the polar coordinate system makes identical digitization networks of the centre symmetrical pictures;

2) corresponding pixels of relevant images are located at the same matrix positions (elements $a_{i j}$ of the same indexes $i$ and $j$ );

3) the central part of the picture (the Moon) can be avoided in the digitization and thus a substantial part of data file is spared (see Fig. 1);

4) quantities at the same distance from the centre of the Sun are placed at one column of the digitization matrix. Similarly, quantities on the same radial are placed at one row;

5) rotation of all the digitized image can be simply performed by shifting of the matrix rows;

6) sector-shaped (Fig. 2) or ring-shaped patterns of the image having explicit topological or physical meanings can be simply processed as sub-matrices of the digitized image.

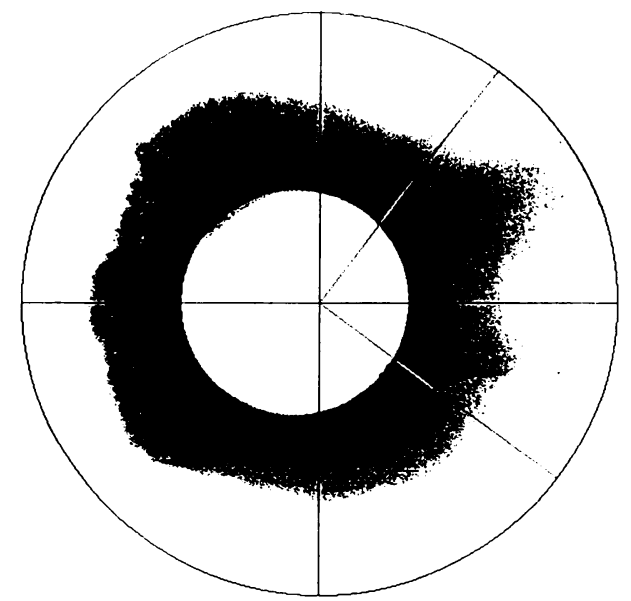

Figure 1. Polarized white-light corona image digitized in the mode of polar coordinate scanning. The eccentricity of the lunar centre to the solar one is intentionally exaggerated. A selected sector is marked by two radials.

There are other special features of the polar scanning mode. Particularly, when the scanning of the image is performed along a radial from $r_{\min }$ to $r_{\max }$, the sampling frequency at each circle of radius $r$ increases as a function of $1 / r$. Thus e.g. for $r_{\min }=0.5 \times r_{\max }$ the corresponding sampling frequency increases 2 times. Some small but systematic shifts of the order of $2 \mu \mathrm{m}$ occur at movement of the microphotometer scanning table along a straight line inclined at a general angle to the axes of a cartesian coordinate system. However, these systematic shifts are negligible when compared to the usually used size of digitisation slit not less than tens of $\mu \mathrm{m}$.

We are convinced that the described facilities of the Universal Microphotometer can also be used to advantage in other astronomical tasks. 

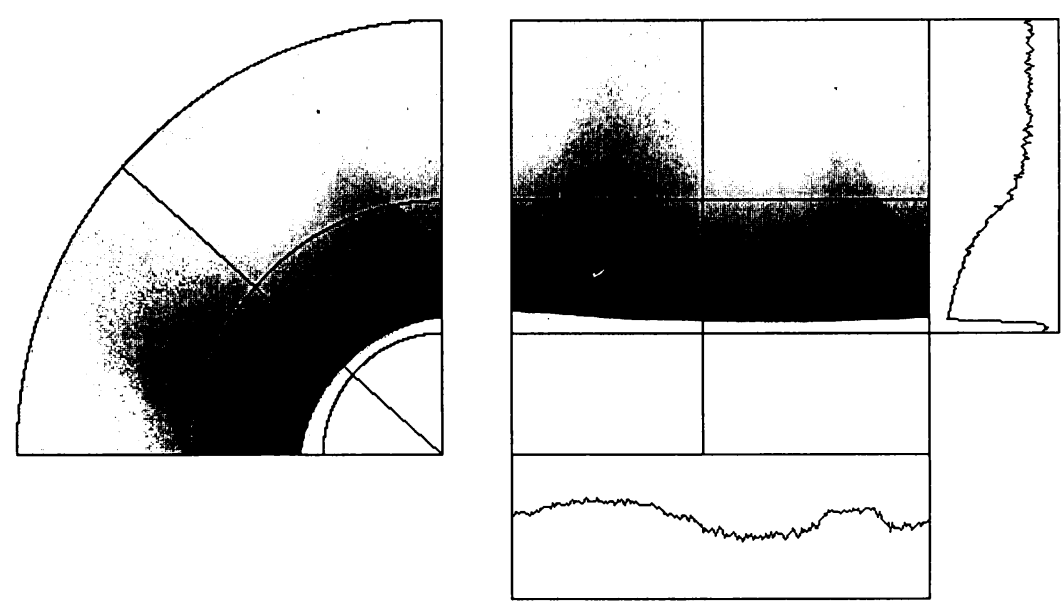

Figure 2. The selected image sector is displayed in the $X$ and $Y$ coordinate system (left), and in the polar coordinate system (right). The light intensity $I$ vs. the polar angle $\varphi$ as well as vs. the coordinate $r$ are shown.

\section{References}

Sykora, J. and Badalyan, O.G., 1992. Proceedings of the first SOHO workshop, Annapolis, U.S.A., 25-28 August 1992, ESA SP-348, November 1992.

Zicha, J., Kotř̌, P., Klvaňa, M. and Knížek, M., 1992. IAU Working Group on Wide-field Imaging, Newsletter No. 2, ed. H.T. MacGillivray, Royal Observatory Edinburgh, p. 72. 